\title{
One-Pot Homologation of Boronic Acids: A Platform for Diversity- Oriented Synthesis
}

\author{
Calum W. Muir, ${ }^{\dagger}$ Julien C. Vantourout, ${ }^{\dagger, \ddagger}$ Albert Isidro-Llobet, ${ }^{\ddagger}$ Simon J. F. Macdonald, \\ and Allan J. B. Watson*, ${ }^{\dagger}$
}

${ }^{\dagger}$ Department of Pure and Applied Chemistry, WestCHEM, University of Strathclyde, 295 Cathedral St., Glasgow, G1 1XL, U.K.

${ }^{\ddagger}$ GlaxoSmithKline, Medicines Research Centre, Gunnels Wood Road, Stevenage, Hertfordshire, SG1 2NY, U.K.

Supporting Information

ABSTRACT: Formal homologation of $\mathrm{sp}^{2}$-hybridized boronic acids is achieved via cross-coupling of boronic acids with conjunctive haloaryl BMIDA components in the presence of a suitably balanced basic phase. The utility of this approach to provide a platform for diversity-oriented synthesis in discovery medicinal chemistry is demonstrated in the context of the synthesis of a series of analogues of a BET bromodomain inhibitor.

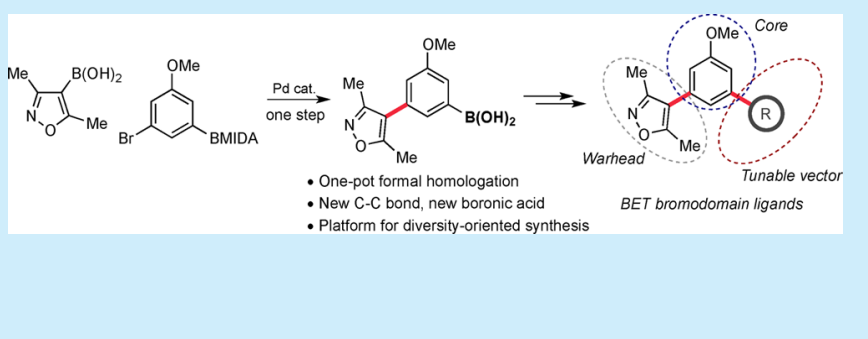

$\mathrm{B}$ oronic acids are among the most versatile of functional groups, capable of a diverse array of chemical reactions. ${ }^{1}$ Their superior reactivity enables many transformations that other standard boron functional groups, such as pinacol boronic (BPin) esters, cannot undergo. ${ }^{2}$ Accordingly, synthetic methods for their preparation are of particular value. Typical preparations involve monoboron transfer processes including the use of stoichiometric organometallic reagents, such as an organolithium or Grignard reagent, with a borate ester and subsequent hydrolysis, ${ }^{1}$ or Pd-catalyzed processes that can directly install the $\mathrm{B}(\mathrm{OH})_{2}$ unit. $^{3}$ However, the majority of catalytic processes using, for example, $\mathrm{Co},{ }^{4} \mathrm{Ir}^{5} \mathrm{Ni}^{6}$ or $\mathrm{Pd}^{7}$ catalysis generate the BPin ester products, which require further processing to access the parent $\mathrm{B}(\mathrm{OH})_{2}$ motif. $^{8}$

Protecting group methods, ${ }^{9-12}$ such as MIDA boronic esters (BMIDA), ${ }^{9}$ have enabled discrimination within differentiated diboron systems, allowing chemoselective cross-coupling of a reactive boronic acid or ester, with a suitable electrophile, to deliver a new $\mathrm{C}-\mathrm{C}$ bond while retaining the BMIDA unit (Scheme 1a). Protecting group removal reveals the latent boronic acid, which can then be further reacted. Alternatively, in situ hydrolysis/Suzuki-Miyaura cross-coupling methods of BMIDA species have been developed. ${ }^{9 \mathrm{i}, \mathrm{k}}$ We have recently shown that the direct generation of BPin esters can be achieved by control of the solution speciation during cross-coupling of monoprotected diboron (BPin/BMIDA) systems (Scheme 1b). ${ }^{13}$ This directly provides a moderately reactive boronic ester product and avoids postreaction protecting group manipulation as well as facilitating the first approach to chemoselective sequential Suzuki-Miyaura cross-coupling using dielectrophile/dinucleophile systems. ${ }^{14,15}$ Based on their increased versatility, direct access to boronic acids via manipulation of diboron systems during cross-coupling would be a valuable addition to the chemical toolbox and would present significant opportunities for the development of
Scheme 1. Suzuki-Miyaura Cross-Coupling with Haloaryl BMIDA Esters: (a) Retention of BMIDA; (b) Generation of BPin; (c) Formal Homologation of Boronic Acids

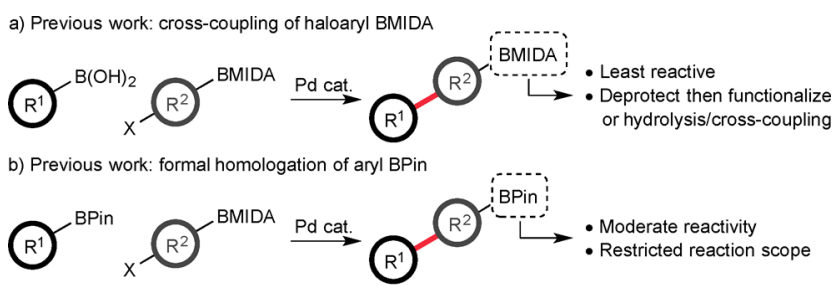

c) This work: formal homologation of aryl boronic acids ........

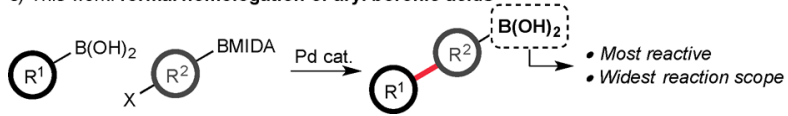

multibond forming tandem reactions. Here we show the development of a formal boronic acid homologation protocol using $\mathrm{B}(\mathrm{OH})_{2} / \mathrm{BMIDA}$ diboron systems (Scheme 1c), enabled by control of the basic phase, and the utility of this process as a platform for diversity-oriented synthesis in the context of epigenetic drug discovery.

We first examined the formal homologation reaction in a benchmark process using $\mathrm{PhB}(\mathrm{OH})_{2}$ (1) and 4-bromophenylboronic acid MIDA ester (2), using a catalyst and medium composition based on our previous work (Table 1, entry 1). ${ }^{13 a, b}$ While good conversion to $3 a$ was recorded, significant protodeboronation was observed. Decreasing the reaction time assisted in moderating this product decomposition pathway but led to incomplete hydrolysis of intermediate 4 (entry 2). Increasing $\mathrm{H}_{2} \mathrm{O}$ loading resolved this problem but again led to increased protodeboronation as well as oligomerization (entry

Received: October 19, 2015

Published: December 3, 2015 
Table 1. Reaction Development ${ }^{a}$

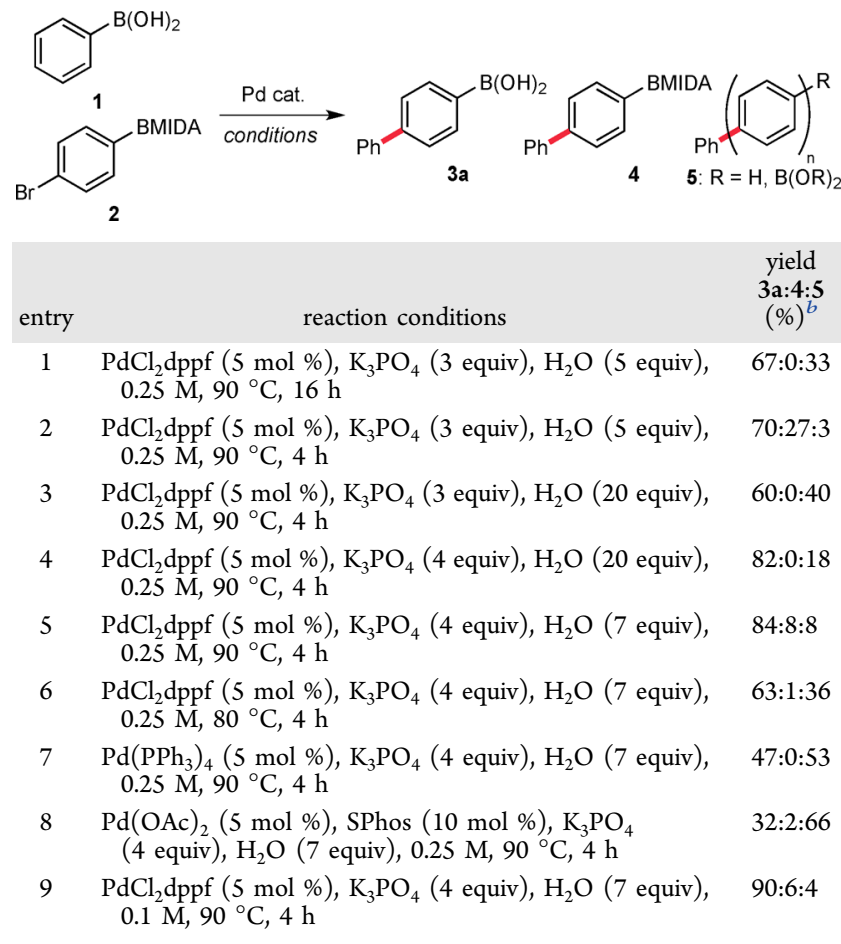

${ }^{a_{1}}$ (1.1 equiv, $0.275 \mathrm{mmol}$ ), 2 ( 1 equiv, $0.25 \mathrm{mmol}$ ), Pd catalyst (5 mol \%), $\mathrm{K}_{3} \mathrm{PO}_{4}, \mathrm{H}_{2} \mathrm{O}, \mathrm{THF}, \mathrm{N}_{2} \cdot{ }^{b}$ Determined by HPLC analysis using an internal standard.

3). Balancing the $\mathrm{H}_{2} \mathrm{O} / \mathrm{K}_{3} \mathrm{PO}_{4}$ composition mitigated these issues (entries 4 and 5). Lowering the reaction temperature (entry 6) and variation of catalyst (entries 7 and 8) were detrimental. However, decreasing the reaction concentration was advantageous, providing $90 \%$ conversion to the desired formal homologation adduct with minimal decomposition (entry 9). These optimized conditions provide a suitable medium for effective cross-coupling ${ }^{14}$ while controlling aqueous base availability to mitigate BMIDA hydrolysis until after the initial $\mathrm{C}-\mathrm{C}$ bond formation has reached completion.

With effective conditions in hand, we moved to establish the generality of the formal homologation process (Scheme 2). The developed protocol operated effectively across a broad range of substrates with variation of the boronic acid component as well as the conjunctive BMIDA unit readily tolerated. The boronic acid component accepted common functional groups including alkyl, amine, ether, thioether, halide, and heterocyclic motifs. Chemoselective cross-coupling was amenable with the bromoaryl BMIDA substrate undergoing oxidative addition over less reactive aryl chloride residues (3o). Notably, both bromovinyl BMIDA and bromoheteroaryl BMIDA components could be employed to deliver the corresponding vinyl ( $3 \mathbf{b}, \mathbf{3 g}$, $3 \mathbf{s})$ and heteroaryl $(4 \mathbf{c}, 3 \mathrm{~d}, 3 \mathbf{i}, 3 \mathbf{3}, 3 \mathbf{r})$ boronic acid products, respectively. Similar species are known to be prone to protodeboronation, but these components performed efficiently in the developed process. ${ }^{9 g, j}$

However, we did observe limitations of the process in the use of heterocyclic 2-boronic acids (benzothiophene, furan, pyridine, and thiophene) as starting materials as well as conjunctive haloheterocyclic 2-BMIDA (e.g., 5-bromothiophene 2-boronic acid MIDA ester) that would generate heterocyclic 2-boronic acid products. In these cases, proto-
Scheme 2. Substrate Scope of the Formal Homologation Reaction $^{a}$
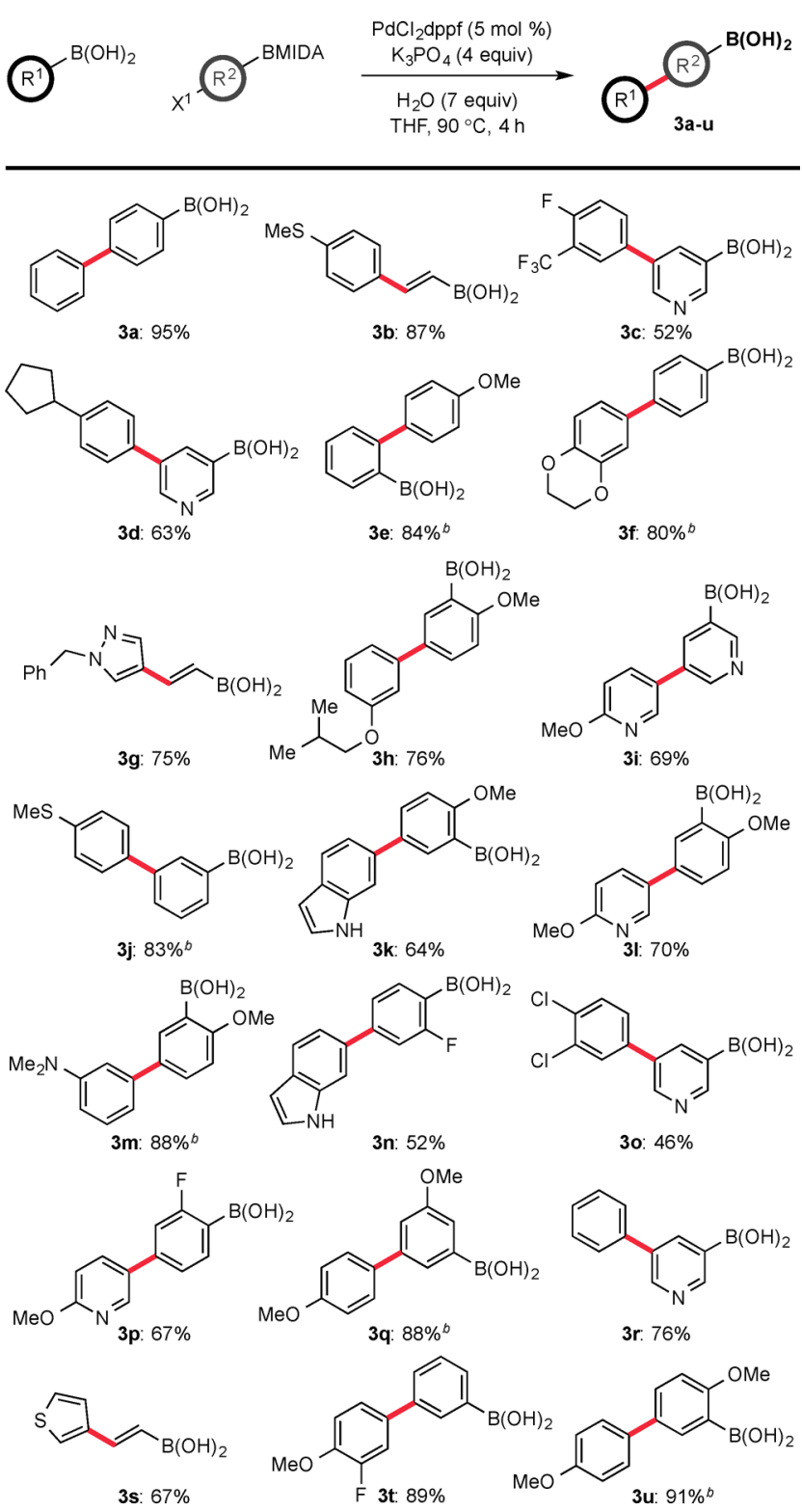

${ }^{a}$ Isolated yields of pure products. ${ }^{b}$ Isolated as the $\mathrm{BF}_{3} \mathrm{~K}$ derivative.

deboronation was found to be significant and the homologation reaction less effective.

The utility of the formal homologation protocol to provide rapid access to valuable and novel chemical space was demonstrated in the context of diversity-oriented synthesis based on a favored pharmacophore that is currently used within epigenetic drug discovery. BET bromodomain inhibitors are targets of particular interest within drug discovery programs. ${ }^{16}$ Compound 6 is an inhibitor of BRD4 and CREBBP (Scheme 3). ${ }^{17}$ The key feature of this molecule is the dimethylisoxazole motif, which acts as an AcK mimic, with the core trisubstituted arene and pendant phenylmethanol providing isoform selectivity and potency modulation vectors. ${ }^{17}$ Installation of the dimethylisoxazole warhead (7) onto the 1,3,5-trisubstituted aryl core (8) using the developed boronic acid homologation protocol provides the key intermediate $3 \mathbf{v}$ in $83 \%$ yield. 
Scheme 3. Formal Homologation of Boronic Acids: A Platform for Diversity-Oriented Synthesis ${ }^{a}$

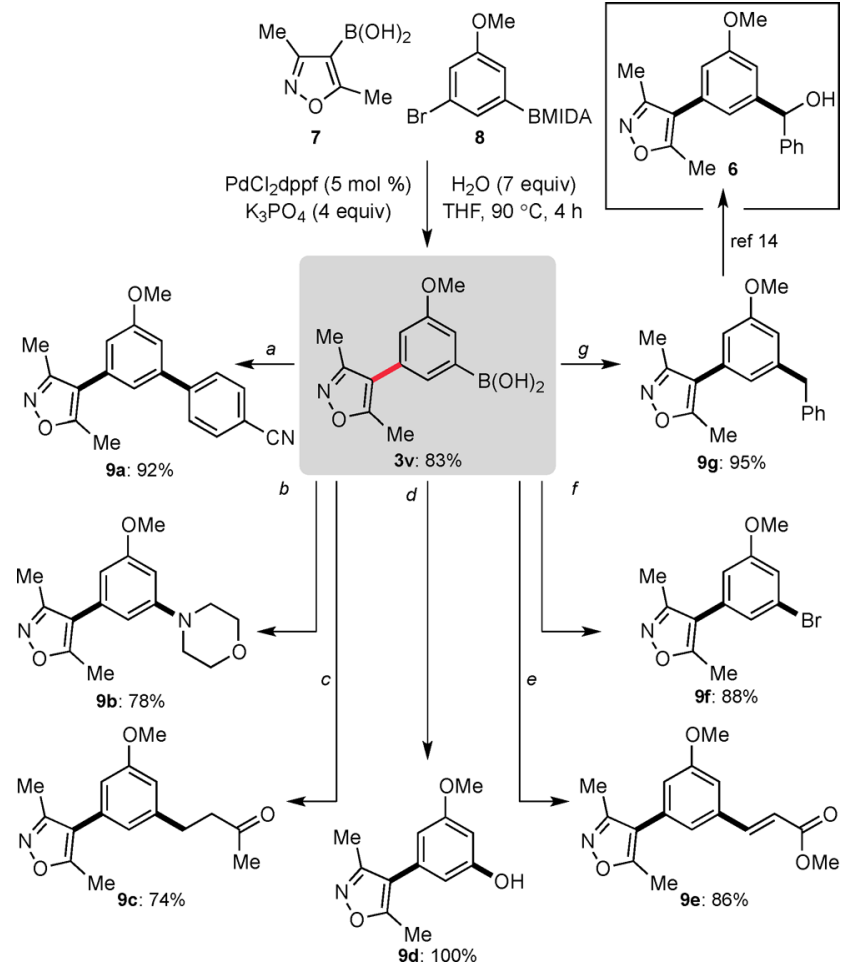

${ }^{a}$ Reaction conditions: (a) $3 \mathbf{v}$ (1.1 equiv), 4-bromobenzonitrile (1 equiv), $\mathrm{PdCl}_{2} \mathrm{dppf}(5 \mathrm{~mol} \%), \mathrm{K}_{3} \mathrm{PO}_{4}$ (3 equiv), $\mathrm{H}_{2} \mathrm{O}$ (5 equiv), THF, $70{ }^{\circ} \mathrm{C}, 1 \mathrm{~h}$; (b) $3 \mathrm{v}$ (1.5 equiv), morpholine (1 equiv), $\mathrm{Cu}(\mathrm{OAc})_{2}(10$ mol \%), MS $4 \AA \mathrm{O}_{2}, \mathrm{CH}_{2} \mathrm{Cl}_{2}, 40^{\circ} \mathrm{C}, 24 \mathrm{~h}$; (c) 3v (1 equiv), methyl vinyl ketone (2 equiv), $[\mathrm{Rh}(\mathrm{COD}) \mathrm{Cl}]_{2}(2 \mathrm{~mol} \%), \mathrm{Na}_{2} \mathrm{CO}_{3}$ (2 equiv), $\mathrm{H}_{2} \mathrm{O}, 80{ }^{\circ} \mathrm{C}, 16 \mathrm{~h}$; (d) $3 \mathbf{v}$ (1 equiv), $\mathrm{H}_{2} \mathrm{O}_{2}$ (10 equiv), THF, rt, $2 \mathrm{~h}$; (e) $3 \mathbf{v}$ ( 1 equiv), methyl acrylate ( 1.5 equiv), 1,4-benzoquinone ( 1 equiv), $\mathrm{Pd}(\mathrm{OAc})_{2}(2 \mathrm{~mol} \%)$, phenanthroline $(2.5 \mathrm{~mol} \%), \mathrm{MeCN}, 60$ ${ }^{\circ} \mathrm{C}, 16 \mathrm{~h}$; (f) $3 \mathrm{v}$ ( 1 equiv), $N$-bromosuccinimide (1.5 equiv), THF, rt, $16 \mathrm{~h}$; (g) 3v (1 equiv), benzyl bromide ( 1.5 equiv), $\mathrm{PdCl}_{2} \mathrm{dppf}(5 \mathrm{~mol}$ $\%), \mathrm{K}_{3} \mathrm{PO}_{4}$ (4 equiv), $\mathrm{H}_{2} \mathrm{O}$ (20 equiv), THF, $90{ }^{\circ} \mathrm{C}, 5 \mathrm{~h}$.

The versatility of the boronic acid motif can then be exploited using established chemistries. For example, further Suzuki-Miyaura reactions using $\mathrm{sp}^{2}$ - and $\mathrm{sp}^{3}$-hybridized electrophiles provided $\mathbf{9 a}$ and $\mathbf{9 g}$, respectively in excellent yield. $9 \mathrm{~g}$ can be readily converted to the parent compound 6 using existing methods. ${ }^{13}$ Chan-Evans-Lam coupling provided the morpholine derivative $9 \mathrm{~b}$ in $78 \%$ yield while Hayashitype 1,4-addition efficiently delivered 9c in $74 \%$ yield. Straightforward Brown-type oxidation to the phenol 9d proceeded quantitatively. An oxidative Heck reaction with methyl acrylate efficiently produced $9 \mathbf{e}$ and, last, the reactivity of the boron-bearing carbon could be inverted by bromination to give 9f. It is important to note that several of these products (e.g., 9b, 9c, 9f) cannot be so readily achieved with either the corresponding BMIDA or BPin derivative of $3 \mathbf{v} ;^{2 a, d}$ these derivatives would require either further processing or alternative, less straightforward chemistries.

In summary, we have developed a boronic acid formal homologation reaction using boronic acids and conjunctive bromoaryl/vinyl BMIDA components. The process relies upon careful control of the basic biphase to enable cross-coupling of haloaryl BMIDA components while avoiding premature hydrolysis. This process gives direct access to functionalized boronic acids that can be used as a platform for diversityoriented synthesis within drug discovery.

\section{ASSOCIATED CONTENT}

Supporting Information

The Supporting Information is available free of charge on the ACS Publications website at DOI: 10.1021/acs.orglett.5b03030.

Experimental procedures, characterization data (PDF)

\section{AUTHOR INFORMATION}

\section{Corresponding Author}

*E-mail: allan.watson.100@strath.ac.uk.

\section{Notes}

The authors declare no competing financial interest.

\section{ACKNOWLEDGMENTS}

This work was supported by the Engineering and Physical Sciences Research Council (EPSRC). We thank the EPSRC UK National Mass Spectrometry Facility at Swansea University for analyses and GlaxoSmithKline for financial and material support.

\section{REFERENCES}

(1) Boronic Acids: Preparation and Applications in Organic Synthesis, Medicine and Materials; Hall, D. G., Ed.; Wiley-VCH: Weinheim, 2011.

(2) For example, BPin esters do not react or are significantly less reactive within the following processes: (a) Frost, C. G.; Penrose, S. D.; Gleave, R. Org. Biomol. Chem. 2008, 6, 4340-4347 (Rh-catalyzed 1,4-addition). (b) Decicco, C. P.; Song, Y.; Evans, D. A. Org. Lett. 2001, 3, 1029-1032 (Cu-catalyzed etherification). (c) Koolmeister, T.; Södergren, M.; Scobie, M. Tetrahedron Lett. 2002, 43, 5965-5968 (Petasis reaction). (d) Chan, D. M. T.; Monaco, K. L.; Li, R.; Bonne, D.; Clark, C. G.; Lam, P. Y. S. Tetrahedron Lett. 2003, 44, 3863-3865 (Cu-catalyzed amination).

(3) (a) Trice, S. L. J.; Dreher, S. D.; Molander, G. A. J. Am. Chem. Soc. 2010, 132, 17701-17703. (b) Kennedy, S. M.; Trice, S. L. J.; Molander, G. A. Org. Lett. 2012, 14, 4814-4817. (c) Tudge, M. T.; Trice, S. L. J.; Kennedy, S. M.; Dreher, S. D.; Molander, G. A. J. Am. Chem. Soc. 2012, 134, 11667-11673.

(4) For example, see: Obligacion, J. V.; Semproni, S. P.; Chirik, P. J. J. Am. Chem. Soc. 2014, 136, 4133-4136.

(5) For examples, see: (a) Cho, J.-Y.; Tse, M. K.; Holmes, D.; Maleczka, R. E.; Smith, M. R. Science 2002, 295, 305-308. (b) Ishiyama, T.; Takagi, J.; Ishida, K.; Miyaura, N.; Anastasi, N. R.; Hartwig, J. F. J. Am. Chem. Soc. 2002, 124, 390-391. (c) Howard, J. A. K.; Peruts, R. N.; Marder, T. B. Angew. Chem., Int. Ed. 2006, 45, 489491. (d) Larsen, M. A.; Hartwig, J. F. J. Am. Chem. Soc. 2014, 136, 4287-4299. (e) Wang, G.; Xu, L.; Li, P. J. Am. Chem. Soc. 2015, 137, $8058-8061$.

(6) For examples, see: (a) Sumida, Y.; Yorimitsu, H.; Oshima, K. J. Org. Chem. 2009, 74, 3196-3198. (b) Yamamoto, T.; Morita, T.; Takagi, J.; Yamakawa, T. Org. Lett. 2011, 13, 5766-5769. (c) Furukawa, T.; Tobisu, M.; Chatani, N. Chem. Commun. 2015, $51,6508-6511$.

(7) For examples, see: (a) Billingsley, K. L.; Barder, T. E.; Buchwald, S. L. Angew. Chem., Int. Ed. 2007, 46, 5359-5363. (b) Billingsley, K. L.; Buchwald, S. L. J. Org. Chem. 2008, 73, 5589-5591. (c) Zhang, L.S.; Chen, G.; Wang, X.; Guo, Q.-Y.; Zhang, X.-S.; Pan, F.; Chen, K.; Shi, Z.-J. Angew. Chem. 2014, 126, 3980-3984. (d) Kawamorita, S.; Ohmiya, H.; Iwai, T.; Sawamura, M. Angew. Chem., Int. Ed. 2011, 50, $8363-8366$

(8) (a) Song, Y.-L.; Morin, C. Synlett 2001, 2, 266-268. (b) Decicco, C. P.; Song, Y.; Evans, D. A. Org. Lett. 2001, 3, 1029-1032. (c) Yuen, A. K. L.; Hutton, C. A. Tetrahedron Lett. 2005, 46, 7899-7903. 
(d) Murphy, J. M.; Tzschucke, C. C.; Hartwig, J. F. Org. Lett. 2007, 9, 757-760.

(9) For examples of the use of BMIDA protecting groups, see: (a) Gillis, E. P.; Burke, M. D. J. Am. Chem. Soc. 2007, 129, 6716-6717. (b) Lee, S. J.; Gray, K. C.; Paek, J. S.; Burke, M. D. J. Am. Chem. Soc. 2008, 130, 466-468. (c) Gillis, E. P.; Burke, M. D. Aldrichimica Acta 2009, 42, 17-27. (d) Woerly, E. M.; Cherney, A. H.; Davis, E. K.; Burke, M. D. J. Am. Chem. Soc. 2010, 132, 6941-6943. (e) Lee, S. J.; Anderson, T. M.; Burke, M. D. Angew. Chem., Int. Ed. 2010, 49, 88608863. (f) Fujii, S.; Chang, S. Y.; Burke, M. D. Angew. Chem., Int. Ed. 2011, 50, 7862-7864. (g) Dick, G. R.; Woerly, E. M.; Burke, M. D. Angew. Chem., Int. Ed. 2012, 51, 2667-2672. (h) He, Z.; Zajdlik, A.; St. Denis, J. D.; Assem, N.; Yudin, A. K. J. Am. Chem. Soc. 2012, 134, 9926-9929. (i) Isley, N. A.; Gallou, F.; Lipshutz, B. H. J. Am. Chem. Soc. 2013, 135, 17707-17710. (j) Woerly, E. M.; Roy, J.; Burke, M. D. Nat. Chem. 2014, 6, 484-491. (k) St. Denis, J. D.; Scully, C. G. C.; Lee, C. F.; Yudin, A. K. Org. Lett. 2014, 16, 1338-1341. (1) Xu, L.; Ding, S.; Li, P. Angew. Chem., Int. Ed. 2014, 53, 1822-1826. (m) St. Denis, J. D.; Zajdlik, A.; Tan, J.; Trinchera, P.; Lee, C. F.; He, Z.; Adachi, S.; Yudin, A. K. J. Am. Chem. Soc. 2014, 136, 17669-17673. (n) Li, J.; Ballmer, S. G.; Gillis, E. P.; Fujii, S.; Schmidt, M. J.; Palazzolo, A. M. E.; Lehmann, J. W.; Morehouse, G. F.; Burke, M. D. Science 2015, 347, 1221-1226. (o) Quiclet-Sire, B.; Zard, S. Z. J. Am. Chem. Soc. 2015, 137, 6762-6765. (p) Li, J.; Grillo, A. S.; Burke, M. D. Acc. Chem. Res. 2015, 48, 2297-2307.

(10) For examples of the use of BDAN protecting groups, see: (a) Noguchi, H.; Hojo, K.; Suginome, M. J. Am. Chem. Soc. 2007, 129, 758-759. (b) Noguchi, H.; Shioda, T.; Chou, C.-M.; Suginome, M. Org. Lett. 2008, 10, 377-380. (c) Iwadate, N.; Suginome, M. Org. Lett. 2009, 11, 1899-1902. (d) Iwadate, N.; Suginome, M. Chem. Lett. 2010, 39, 558-560. (e) Iwadate, N.; Suginome, M. J. Am. Chem. Soc. 2010, 132, 2548-2549. (f) Lee, J. C. H.; McDonald, R.; Hall, D. G. Nat. Chem. 2011, 3, 894-899. (g) Feng, X.; Jeon, H.; Yun, J. Angew. Chem., Int. Ed. 2013, 52, 3989-3992. (h) Xu, L.; Li, P. Chem. Commun. 2015, 51, 5656-5659.

(11) For $\mathrm{BF}_{3} \mathrm{~K}$ as protecting group, see: Molander, G.; Sandrock, D. L. J. Am. Chem. Soc. 2008, 130, 15792-15793.

(12) For reviews of diboron systems involving protecting groups, see: (a) Tobisu, M.; Chatani, N. Angew. Chem., Int. Ed. 2009, 48, 35653568. (b) Wang, C.; Glorius, F. Angew. Chem., Int. Ed. 2009, 48, 52405244. (c) Xu, L.; Li, P. Synlett 2014, 25, 1799-1802. (d) Fyfe, J. W. B.; Watson, A. J. B. Synlett 2015, 26, 1139-1144.

(13) (a) Fyfe, J. W. B.; Seath, C. P.; Watson, A. J. B. Angew. Chem., Int. Ed. 2014, 53, 12077-12080. (b) Fyfe, J. W. B.; Valverde, E.; Seath, C. P.; Kennedy, A. R.; Anderson, N. A.; Redmond, J. M.; Watson, A. J. B. Chem. - Eur. J. 2015, 21, 8951-8964. See also: (c) Molloy, J. J.; Law, R. P.; Fyfe, J. W. B.; Seath, C. P.; Hirst, D. J.; Watson, A. J. B. Org. Biomol. Chem. 2015, 13, 3093-3102.

(14) Seath, C. P.; Fyfe, J. W. B.; Molloy, J. J.; Watson, A. J. B. Angew. Chem., Int. Ed. 2015, 54, 9976-9979.

(15) For the recent development of a dual catalysis method to achieve chemoselective cross-coupling of diboron systems without protecting groups, see: Yamashita, Y.; Tellis, J. C.; Molander, G. A. Proc. Natl. Acad. Sci. U. S. A. 2015, 112, 12026-12029.

(16) Filippakopoulos, P.; Knapp, S. Nat. Rev. Drug Discovery 2014, 13, 337-356.

(17) Hewings, D. S.; Fedorov, O.; Filippakopoulos, P.; Martin, S.; Picaud, S.; Tumber, A.; Wells, C.; Olcina, M. M.; Freeman, K.; Gill, A.; Ritchie, A. J.; Sheppard, D. W.; Russell, A. J.; Hammond, E. M.; Knapp, S.; Brennan, P. E.; Conway, S. J. J. Med. Chem. 2013, 56, 3217-3227. 